\title{
El rol de la tecnología en el desarrollo de entendimiento matemático vía la resolución de problemas
}

\section{The role of technology in the development of mathematical understanding through problem solving}

\author{
Fernando Barrera-Mora ${ }^{1}$ \\ fbarrera10147@gmail.com, \\ Aarón Reyes-Rodríguez \\ aaron.reyes.rdz@gmail.com, \\ Universidad Autónoma del Estado de Hidalgo, México
}

\section{Resumen:}

El uso de tecnologías digitales ha llevado a incorporar nuevos elementos de reflexión para abordar el problema de aprendizaje matemático, ya que al resolver problemas con tecnologías digitales aparecen elementos que en un ambiente de papel y lápiz pueden quedar ocultos. Por ejemplo, al describir el cambio de volumen al variar la altura de un líquido en un recipiente, el estudiante debe imaginar cómo se da ese cambio. Sin embargo, con el uso de la tecnología, puede visualizar tal cambio y concentrar la atención en otros aspectos, tales como la razón de cambio o la convidad de la gráfica. En este trabajo presentamos resultados de un estudio que involucró el uso de GeoGebra para desarrollar tareas de instrucción enfocadas a explorar el entendimiento matemático de los estudiantes para formular relaciones funcionales y su

\begin{abstract}
:
The use of digital technologies has incorporated new reflection elements to approach the problem of mathematics learning since these tools can uncover elements that could be hidden when students use pencil and paper to solve problems. For instance, to describe how the volume of a liquid in a container changes when the height of that liquid is modified requires the student to imagine how the change occurs. However, with the use of digital technologies, the student can visualize such variation and focus his attention on other important aspects such as the rates of change or the way in which that change occurs. In this paper we report some results from a study conducted with a group of students that used GeoGebra to approach mathematical tasks aimed at exploring students' understanding regarding functional relations
\end{abstract}

1 Dirección para correspondencia (correspondence address):

Fernando Barrera Mora. Área Académica de Matemáticas y Física, Instituto de Ciencias Básicas e Ingeniería de la Universidad Autónoma del Estado de Hidalgo. Ciudad del Conocimiento de la UAEH, Carretera Pachuca Tulancingo Km 4.5, Colonia Carboneras, CP 42184 (México). 
El rol de la tecnología en el desarrollo de entendimiento matemático vía la resolución de problemas

Fernando Barrera-Mora y Aarón Reyes-Rodríguez

implicación al elaborar gráficas de volumen vs altura. Las tareas se implementaron con estudiantes de primer semestre de una licenciatura en matemáticas, de una universidad pública en México. La información se analizó desde una perspectiva epistemológica socioconstructivista y una posición didáctica que privilegia el entendimiento matemático, vía la resolución de problemas. Las unidades de análisis fueron las secuencias de razonamiento desarrolladas por los estudiantes. El uso sistemático de tecnología promovió la formación de estructuras conceptuales en procesos de razonamiento de tipo cualitativo y cuantitativo. También se identificaron algunas limitaciones de la herramienta para promover la organización de ideas, sobre todo aquellas que se refieren a la concavidad de las gráficas.

\section{Palabras clave:}

Tecnología digital; resolución de problemas; entendimiento; representaciones gráficas.

\section{Résumé:}

L'usage des technologies digitales a incité à incorporer de nouveaux éléments de réflexion pour aborder le problème de l'apprentissage mathématique car, en résolvant des problèmes avec les technologies digitales, on découvre des éléments qui peuvent rester cachés quand on utilise seulement du papier et un crayon. Par exemple, en décrivant le changement de volume après la variation du niveau d'un liquide dans un récipient, l'étudiant doit imaginer comment ce changement arrive. Néanmoins, avec l'usage de la technologie, l'étudiant peut visualiser ce changement et concentrer son attention sur $d^{\prime}$ autres aspects comme la raison du changement ou la concavité du graphique. Dans ce travail, nous présentons des résultats d'une étude qui inclus l'usage de GeoGebra pour développer des tâches d'instruction dirigées à explorer la compréhension mathématique des étudiants pour formuler des relations fonctionnelles et leur implication dans l'élaboration des graphiques de volume et niveau. Les tâches sont exécutées avec des étudiants de premier semestre de la licence en mathématiques d'une université publique du Mexique. L'information est analysée selon une perspective épistémologique socioconstructiviste et une position didactique qui privilégie la compréhension mathématique via la résolution des problèmes. Les unités d'analyse sont les séquences de raisonnements développés par les étudiants. L'usage systématique de la technologie a promu la formation des structures conceptuelles dans un processus de raisonnement de type qualitatif et quantitatif. Aussi, quelques limitations de l'instrument pour promouvoir l'organisation des idées sont identifiées, surtout celles qui font référence à la concavité des graphiques.

Mots clés:

Technologie digitale; résolution de problèmes; compréhension; représentations graphiques.

Fecha de recepción: 9-5-2018

Fecha de aceptación: 24-9-2018 


\section{Introducción}

Resolver problemas con el uso de tecnologías digitales puede promover formas de razonar que difieren de aquellas que se llevan a cabo al utilizar otras herramientas, como papel y lápiz. Esto podría deberse a que cualquier actividad cognitiva es una actividad mediada por las herramientas que se utilizan (Werstch, 1993). Por otro lado, desde una perspectiva sociocultural, el conocimiento humano se considera un producto de la interacción entre nuestras estructuras mentales y las acciones que desarrollamos con los artefactos o tecnologías, materiales o simbólicas, disponibles en un entorno sociocultural (Vygotsky, 1962, 1978). También se tiene que las herramientas influyen en las formas de pensar y razonar, ya que palabras, textos, símbolos, gráficas y tablas, nos permiten representar y organizar ideas, además de reflexionar sobre los procesos de pensamiento de formas particulares que desarrollamos (Pea, 1987; Koehler y Mishra, 2009). Así, las representaciones se constituyen en recursos de mediación para el entendimiento y las diversas maneras de construir conocimiento matemático (Moreno-Armella y Santos-Trigo, 2008).

Las tecnologías materiales o simbólicas modifican nuestras estructuras mentales, al amplificar y reorganizar nuestros recursos cognitivos, e integrarse estrechamente con ellos (Moreno-Armella y Santos-Trigo, 2008). Por ejemplo, la escritura es una herramienta que permite, por una parte, trascender las limitaciones biológicas de la memoria (recordar una gran cantidad de información que podemos organizar para llevar a cabo tareas importantes de nuestras actividades cotidianas) y por otra, reflexionar sobre ideas y procesos de pensamiento, con la finalidad de refinarlos o modificarlos. Esto es, la capacidad de escribir transforma la arquitectura funcional del cerebro y tiene un profundo impacto en cómo desarrollamos la actividad cognitiva (Donald, 2001). Otro ejemplo de tecnología cognitiva es el sistema de numeración decimal, cuyo uso moldea las capacidades para realizar cálculos (Kaput y Schorr, 2008, p. 212).

Las representaciones generadas en un ambiente digital se caracterizan por la posibilidad que tiene un usuario de relacionarlas entre sí de forma dinámica, de modo que un cambio en una de ellas se vea reflejado, en tiempo real, en las otras representaciones con las que está ligada, manteniéndose invariante cierto conjunto de relaciones estructurales 
El rol de la tecnología en el desarrollo de entendimiento matemático vía la resolución de problemas

Fernando Barrera-Mora y Aarón Reyes-Rodríguez

(Moreno-Armella, 2002). En este sentido, se dice que las representaciones computacionales, particularmente de un software como GeoGebra, son representaciones ejecutables (Moreno-Armella, Hegedus y Kaput, 2008). Se entiende por representaciones ejecutables aquellas que externalizan funciones cognitivas algorítmicas que anteriormente eran del dominio exclusivo de los seres humanos. Por ejemplo, revisar la ortografía de una palabra; trazar una gráfica a partir de una ecuación; operar representaciones algebraicas para encontrar las raíces de una ecuación o para obtener derivadas e integrales de funciones; operar con números reales o complejos, entre otras. Otra característica de estas representaciones es que permiten visualizar acciones que antes sólo ocurrían en la imaginación, como la realización de un zoom sobre una gráfica, trazar el lugar geométrico que describe un punto, o aplicar una transformación rígida a un objeto geométrico (Moreno-Armella y Santos-Trigo, 2008).

Dada la relevancia de las tecnologías digitales como amplificadores y reorganizadores de la cognición, resulta importante reflexionar acerca de posibles transformaciones de los ambientes de aprendizaje, con la finalidad de aprovechar las ventajas que ofrece el uso de estas tecnologías (Santos-Trigo y Moreno-Armella, 2016). Algunas interrogantes en las que estamos interesados son: ¿Cuáles son las características del conocimiento que se construye al resolver problemas con GeoGebra? ¿Cómo el uso de GeoGebra, durante la resolución de problemas, puede apoyar el desarrollo de entendimiento matemático? ¿Qué formas de razonamiento emergen como resultado de usar GeoGebra para resolver problemas?

Como se mencionó previamente, el conocimiento humano es producto de la interacción entre las estructuras mentales y las acciones que se desarrollan con los artefactos o tecnologías al resolver problemas. Por otra parte, el aprendizaje es el conocimiento o habilidades que se construyen durante procesos de instrucción o estudio. En este trabajo nos interesa identificar algunos elementos que pueden ayudar a que estudiantes de primer semestre, de una licenciatura en matemáticas aplicadas, desarrollen un aprendizaje con entendimiento (Hiebert et al., 1997) del concepto de función, cuando se les ofrecen oportunidades para resolver tareas con el uso de herramientas digitales. De acuerdo con Hiebert et al. (1997), entender algo significa, mediante procesos de reflexión y comunicación de ideas, relacionar o conectar ese algo con otras cosas que se han aprendido previamente. De esta conceptualización de entendimiento, en términos de conexiones, se desprende que pueden existir diversos 
niveles de éste, en función de la cantidad y robustez de las relaciones que se establecen entre una idea y otros conocimientos previos (Sarama y Clements, 2009; Chimhande, Naidoo y Stols, 2017). Así, hemos aprendido algo con entendimiento si podemos conectar o relacionar ese algo, de forma significativa, con otras cosas que conocemos.

Particularmente, buscamos reconocer niveles de entendimiento del concepto de función desarrollados por estudiantes de primer semestre de licenciatura, al abordar actividades de llenado de recipientes, en las que deben representar el volumen de un líquido como función de la altura que éste alcanza en el recipiente. Es decir, se pretende identificar qué conexiones establecen entre ideas relativas al concepto de función, al abordar tareas con el uso de GeoGebra, y el posible impacto de la herramienta en la construcción de tales conexiones. Dado que no se tiene un acceso directo al pensamiento de los estudiantes, la evidencia del entendimiento se obtiene de las justificaciones que elaboran y comunican durante los procesos de razonamiento. Finalmente, entendemos por razonamiento una trayectoria de pensamiento desarrollada para producir afirmaciones, justificarlas y alcanzar conclusiones al resolver una tarea (Lithner, 2008). Las tareas se orientaron al análisis de modelos dinámicos, elaborados con GeoGebra, enfocando la atención en la construcción de gráficas de volumen vs altura. Esto tiene como antecedente diversas investigaciones que han aportado evidencia de las dificultades que exhiben estudiantes, con un extenso bagaje matemático, al realizar tareas que involucran graficar funciones (Carlson et al., 2002; Heid et al., 2006).

\section{Tareas de llenado de recipientes y el concepto de función}

La investigación en torno a la elaboración de representaciones gráficas de funciones, y su relación con un fenómeno físico o una representación (dibujo, fotografía o modelo dinámico de un recipiente), se ha orientado en diversas direcciones. En relación con experiencias al Ilenar recipientes, se ha investigado la forma en que estudiantes o profesores representan gráficas de área superficial vs altura, volumen vs altura, altura vs volumen, o altura vs área superficial, considerando recipientes de diferentes formas sin el uso de un modelo algebraico (SCME, 1985; HittEspinosa, 1995; Carlson et al., 2002; Carlson, Larsen y Lesh, 2003; De 
El rol de la tecnología en el desarrollo de entendimiento matemático vía la resolución de problemas

Fernando Barrera-Mora y Aarón Reyes-RodríGuez

Faria-Campos, 2004; Heid, et al., 2006; Johnson, 2012; de Beer, Gravemeijer y van Eijck, 2015). En la mayoría de las investigaciones se muestra a los participantes dibujos o fotografías de recipientes, pidiéndoles imaginar el proceso de llenado y, posteriormente, bosquejar la gráfica que representa la relación entre ciertas variables. En otros casos, las tareas consisten en determinar la forma del recipiente dada la gráfica de la función.

En una investigación realizada por Hitt-Espinosa (1995), se pidió a profesores en servicio bosquejar gráficas de volumen vs altura, a partir de dibujos de recipientes con diversas formas. Se identificaron respuestas correctas e incorrectas, y el posible origen de los errores, concluyendo que la forma del recipiente influyó en que los participantes no elaboraran correctamente las gráficas, ya que contornos redondos o lineales de los recipientes indujeron el bosquejo de gráficas con formas semejantes (fenómeno de traslación de formas). Además, se observó una preeminencia de las funciones continuas sobre las discontinuas, como en el caso de errores cometidos al graficar el área superficial vs altura, para un recipiente parecido a un pastel de cumpleaños. Análogamente, De Faria-Campos (2004) exploró los errores que cometen profesores en formación, al elaborar gráficas de área vs altura. El autor resalta que los errores se deben, principalmente, a la traslación de formas y la tendencia de los participantes a buscar, en primer término, representaciones algebraicas de las funciones y a partir de éstas bosquejar las gráficas respectivas.

Otras investigaciones se han enfocado en explorar la utilidad de la razón instantánea de cambio como una herramienta que permite determinar y entender la forma de gráficas de altura vs volumen. Al respecto, Carlson y colaboradores (2002) identificaron niveles progresivos de razonamiento covariacional. Al aplicar estos niveles para analizar el trabajo de estudiantes de licenciatura, con alto desempeño en la asignatura de cálculo, los autores observaron que sólo el $25 \%$ de los participantes construyó una gráfica aceptable, además de que pocos estudiantes fueron capaces de formarse imágenes mentales precisas de la tasa instantánea de cambio; particularmente, para distinguir tasas crecientes o decrecientes. En esta misma línea de ideas, Carlson, Larsen y Lesh (2003) pidieron a profesores explicar, verbalmente, por qué gráficas de altura vs volumen son suaves y cómo se relacionan los cambios en la gráfica con la forma del recipiente. Todos los participantes produjeron 
gráficas razonables, debido a que se les solicitó explícitamente refinar la solución, considerando retroalimentación que recibieron de sus compañeros después de verbalizar sus procesos de razonamiento. Sin embargo, aparecieron algunas dificultades cuando se les pidió graficar la altura de un líquido en una botella como función del volumen. Entre estas dificultades destaca la consideración de la altura o el tiempo como variables, y no el volumen, como se solicitaba en el problema. También se identificó una ausencia de reflexión sobre cómo obtener las gráficas, punto por punto, o cómo refinar segmentos de línea que representan tasas promedio de cambio.

Johnson (2012) utilizó fotografías de botellas de diversas bebidas y pidió a los estudiantes graficar altura vs volumen y viceversa, con la finalidad de identificar formas en que estudiantes de secundaria dan sentido a la tasa de cambio, y conocer como desarrollan habilidades de razonamiento covariacional. En un trabajo posterior, este autor adaptó el problema de llenado de recipientes al considerar "llenado" de polígonos (Johnson, 2013). Se diseñó una secuencia de tareas que involucraron elaborar gráficas de área vs altura y el uso del software The Geometer Sketchpad, para hacer manejable el problema a estudiantes de séptimo grado, de modo que la progresión en el uso de razonamientos cualitativos de los estudiantes, apoyara la construcción de coordinaciones entre cantidades que varían de forma conjunta. Uno de los aportes del trabajo fue la identificación de un conjunto de consideraciones teóricas, incluyendo caracterización de procesos mentales que emergen al dar sentido y coordinar cantidades covariantes, y consideraciones prácticas, entre las que destacan la forma de operar de los estudiantes con cantidades representadas en configuraciones dinámicas.

Respecto de las investigaciones que han utilizado herramientas digitales, de Beer (2015) utilizó dibujos de copas, con el objetivo de promover, en estudiantes de primaria, un entendimiento cualitativo de la tasa de cambio, a través del uso e interpretación de gráficas de volumen vs altura. Se observó que los estudiantes describieron el proceso de cambio mediante dibujos que representan instantáneas del proceso de llenado, y mediante estas representaciones, notaron que la tasa de cambio depende de la anchura de la sección transversal del recipiente. Los estudiantes elaboraron inicialmente una gráfica de barras y sólo con apoyo del instructor y una herramienta tecnológica, lograron construir una curva continua como representación que describe el Ilenado de la copa. En otros 
El rol de la tecnología en el desarrollo de entendimiento matemático vía la resolución de problemas

Fernando Barrera-Mora y Aarón Reyes-RodríGuez

trabajos se utilizaron copas de diferentes tipos y un vaso jaibolero (de Beer, 2017). Los estudiantes no tuvieron dificultad para razonar acerca de la tasa de cambio constante implicada en el Ilenado del vaso jaibolero. Sin embargo, analizar el llenado de una copa comparando secciones transversales de ésta con la del vaso, permitió determinar la naturaleza no lineal de la función que modela el proceso de llenado de la copa. Además, los estudiantes relacionaron cada instantánea con un punto de la gráfica discreta que construyeron para representar la relación entre altura y volumen.

Con base en la revisión de la literatura, se identificó que la mayoría de las investigaciones se estructuran en torno de tareas desarrolladas con papel y lápiz, y solo en algunos casos se incluyó el uso de tecnologías digitales (Johnson, 2013; Thomson, 2013; de Beer, 2017), por lo que algunos investigadores sugieren realizar trabajos que analicen el impacto de las tecnologías digitales en el entendimiento del concepto de función (Hitt-Espinosa, 1995; De Faria-Campos, 2004). Con estos antecedentes, buscamos aportar elementos que permitan identificar el papel que juegan las configuraciones dinámicas, elaboradas con GeoGebra, en la construcción de entendimiento matemático durante el proceso de elaboración de gráficas que modelan el Ilenado de recipientes.

\section{Naturaleza de la matemática, su aprendizaje y su entendimiento}

Investigaciones recientes en educación matemática han mostrado que los programas de investigación se estructuran en torno a más de una perspectiva, esto con la finalidad de analizar, con más elementos teóricos, los procesos cognitivos e identificar las dificultades que muestran los estudiantes cuando incorporan a la tecnología al abordar tareas de aprendizaje matemático. El uso de diversas perspectivas teóricas tiene como finalidad elaborar bases robustas que permitan enmarcar adecuadamente el uso de artefactos digitales en el salón de clase (Mishra y Koehler, 2006). Al respecto, el marco de investigación que orienta este trabajo incluye tres dimensiones: (i) ontológica, (ii) epistemológica y (iii) didáctica.

En cuanto a la dimensión ontológica, consideramos que la matemática es la ciencia de los patrones (Steen, 1988) y que aprender matemáticas consiste, en gran medida, en adquirir una disposición para ver el 
mundo a través de la lente de un matemático (Schoenfeld, 1992). Esta disposición incluye llevar a cabo actividades entre las que destacan: experimentar, explorar relaciones matemáticas, formular conjeturas, justificar resultados, comunicar ideas, así como resolver problemas por diferentes rutas (Polya, 1945) y desarrollar una actitud inquisitiva; esto es, habilidad para formular, de manera sistemática, preguntas y nuevos problemas (Berger, 2014; Santos-Trigo, 2018).

En cuanto a la dimensión epistemológica, adoptamos una perspectiva de corte socio constructivista (Simon, 1994), por lo cual suponemos, por un lado, que cada persona construye, de forma activa, su propio conocimiento al abordar problemas que desequilibran sus estructuras cognitivas, independientemente del contexto o la presencia y naturaleza del proceso de enseñanza. Por otro lado, consideramos que el aprendizaje es un proceso que se lleva a cabo en una comunidad donde se construyen significados o entendimientos considerados como compartidos (Cobb et al., 1991). Dado que aprender es un proceso social, el medio cultural y sus producciones determinan las características del conocimiento que construimos (Werstch, 1991). Particularmente, la naturaleza de la actividad cognitiva se encuentra estrechamente ligada a la generación y uso de representaciones semióticas (Moreno-Armella y Hegedus, 2009, p. 501), esto debido a que estas estructuras simbólicas constituyen un medio que nos permiten actuar sobre el mundo pero, a su vez, imponen regulaciones a nuestro pensamiento acerca del mundo (MorenoArmella, Hegedus y Kaput, 2008; Santos-Trigo y Moreno-Armella, 2016).

La dimensión didáctica se refiere a las características del aprendizaje que consideramos deseables. Estas características tienen como eje un conocimiento altamente estructurado. Particularmente, en este trabajo estamos interesados en que los estudiantes desarrollen entendimiento de las ideas matemáticas (Hiebert, et al. 1997), lo que implica la construcción de conexiones robustas entre un conocimiento nuevo y conocimientos previos, a partir de procesos de reflexión y comunicación de ideas, que se llevan a cabo durante la resolución de problemas. Dado que no se puede tener un acceso directo al pensamiento de los estudiantes, la evidencia del entendimiento se obtiene de las justificaciones que llevan a cabo, y que están inmersas en las comunicaciones o producciones (escritas, verbales, gestuales, icónicas) que externan. Por otra parte, consideramos que el razonamiento es una trayectoria de pensamiento desarrollada para producir afirmaciones, justificarlas y obtener conclu- 
El rol de la tecnología en el desarrollo de entendimiento matemático vía la resolución de problemas

Fernando Barrera-Mora y Aarón Reyes-Rodríguez

siones al resolver una tarea (Lithner, 2008). Una secuencia de razonamiento se puede pensar como una gráfica dirigida (Figura 1) en la que un vértice representa tanto un estado momentáneo de conocimiento como una sub-tarea. Cada arista de la gráfica representa la implementación de una estrategia que implica el tránsito desde un estado de conocimiento a otro. Una razón es la justificación que sustenta la transición entre dos vértices de la gráfica entre los cuales existe una arista (Reyes-Rodríguez, Vargas-Alejo, Cristobal-Escalante y Soberanis-Cruz, 2015). En la Figura 1 se ilustra una secuencia de razonamiento para una tarea que tiene dos respuestas (vértices 4 y 8 ), una de las cuales (la respuesta 1 ) se puede obtener por dos caminos diferentes.

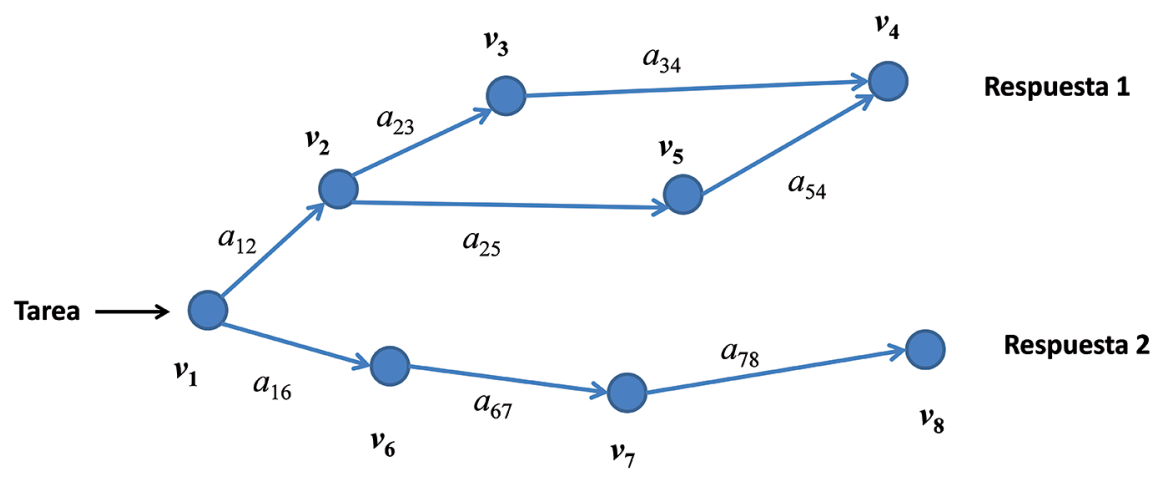

Figura 1. Ilustración de una secuencia de razonamiento (adaptado de Lithner, 2008).

Adoptamos una aproximación a la resolución de problemas basada en proponer actividades de modelización que brinden oportunidades a los estudiantes para desarrollar aspectos esenciales del pensamiento matemático (explorar relaciones, formular conjeturas, comunicar resultados, plantear preguntas o interrogantes). Lo anterior se relaciona estrechamente con la promoción de un ambiente de instrucción en el que los estudiantes trabajan en pequeños grupos, y realizan presentaciones plenarias del proceso de solución de los problemas. También se requiere soporte instruccional sustantivo por parte del profesor para guiar las actividades, entre los que destaca la promoción de procesos de reflexión y comunicación de ideas (Santos-Trigo, 2014).

Con la finalidad de identificar posibles niveles de entendimiento del concepto de función utilizamos el marco de pensamiento covariacional 
propuesto por Carlson, Larsen y Lesh (2003). Este marco describe seis categorías de acciones mentales que se han observado en estudiantes cuando representan e interpretan modelos gráficos de fenómenos modelados por funciones. Es importante notar que en los procesos de razonamiento que llevan a cabo los estudiantes, no necesariamente están involucradas todas las acciones mentales del marco, las cuales no están ordenadas secuencialmente. Las categorías de acciones mentales son las siguientes: (1) una imagen de dos variables que cambian simultáneamente, (2) una imagen coordinada general de cómo las variables cambian, una respecto de otra (crece o decrece), (3) una imagen del cambio de la función para incrementos fijos de la variable, (4) una imagen de la tasa de cambio para intervalos, (5) una imagen de la tasa de cambio instantánea en todo el dominio de la función y (6) una imagen de los intervalos donde la tasa de cambio es creciente o decreciente. Consideramos que los estudiantes tienen un nivel de entendimiento más robusto entre más elementos del marco incorporen en sus respuestas o explicaciones al llevar a cabo las tareas propuestas.

\section{Metodología}

Las actividades se implementaron con un grupo de 18 estudiantes de primer semestre de una licenciatura en matemáticas aplicadas, en una universidad pública de México. Todos los estudiantes habían cursado, durante el bachillerato (grados 10-12), las asignaturas de álgebra, geometría analítica y cálculo. Es importante mencionar que, en general, los cursos de cálculo de bachillerato en México tienen un enfoque algorítmico. En estos cursos se enfatiza muy poco el entendimiento de la derivada como tasa instantánea de cambio. Solamente se menciona a los estudiantes que la derivada es una tasa de cambio instantánea, sin profundizar esta idea.

Durante el semestre académico en el que se llevó a cabo la investigación, los estudiantes se encontraban inscritos en cuatro cursos de matemáticas de licenciatura: (i) computación y matemáticas, (ii) geometría, (iii) cálculo elemental y (iv) razonamiento matemático. El curso donde se recolectó la información estuvo orientado a la resolución de problemas con la utilización de diversas tecnologías digitales, como Excel, GeoGebra y Derive, y estuvo a cargo de uno de los investigadores, 
El rol de la tecnología en el desarrollo de entendimiento matemático vía la resolución de problemas

Fernando Barrera-Mora y Aarón Reyes-Rodríguez

quien también era el profesor regular del curso. Durante las sesiones de trabajo se promovió el desarrollo de diversos aspectos del pensamiento matemático, mediante la resolución de problemas en pequeños grupos, así como la exposición plenaria de los resultados. Las presentaciones plenarias se videograbaron y posteriormente se transcribieron las grabaciones. Además, se recolectaron las producciones escritas y los archivos de GeoGebra que elaboraron los participantes.

Las tareas involucraron el análisis del cambio de volumen de un líquido, contenido en recipientes de distintas formas (cilíndricas, semiesféricas y cónicas), como función de la altura, a partir de modelos dinámicos, elaborados por el grupo de investigación, con el software GeoGebra. Durante tres sesiones, de dos horas cada una, el instructor proporcionó apoyo y retroalimentación a los estudiantes para la realización de las tareas. Durante estas sesiones no se revisaron fórmulas para el cálculo de volúmenes. Las preguntas que guiaron la recolección de información fueron las siguientes: ¿Cuáles son las cantidades que varían? ¿Cuál es la relación entre esas variaciones? ¿Cuál es el volumen del líquido para valores específicos de la altura? ¿Dónde cambia más rápido el volumen?

En una cuarta sesión, se entregó a cada equipo, conformado por tres estudiantes, una configuración dinámica que simulaba el Ilenado de un recipiente, con la finalidad de que los estudiantes bosquejaran gráficas de volumen vs altura, sin ayuda ni retroalimentación del instructor. Esta tarea tuvo la finalidad de conocer cómo el uso de la herramienta puede apoyar la construcción de conexiones entre ideas relacionadas con el concepto de función, así como la identificación de posibles limitaciones de la herramienta o del escenario de instrucción en la construcción de entendimiento respecto de las representaciones gráficas. Se seleccionaron las tareas de la esfera y el cono invertido, de modo que los estudiantes pudieran identificar semejanzas y diferencias con las tareas en las que recibieron apoyo y retroalimentación del instructor. Tres equipos analizaron la configuración dinámica que modela el llenado de una esfera completa y otros tres equipos una configuración dinámica que modela el llenado de un cono invertido, cuyas gráficas de volumen vs altura se muestran en la Figura 2. La asignación de las tareas se hizo aleatoriamente. 


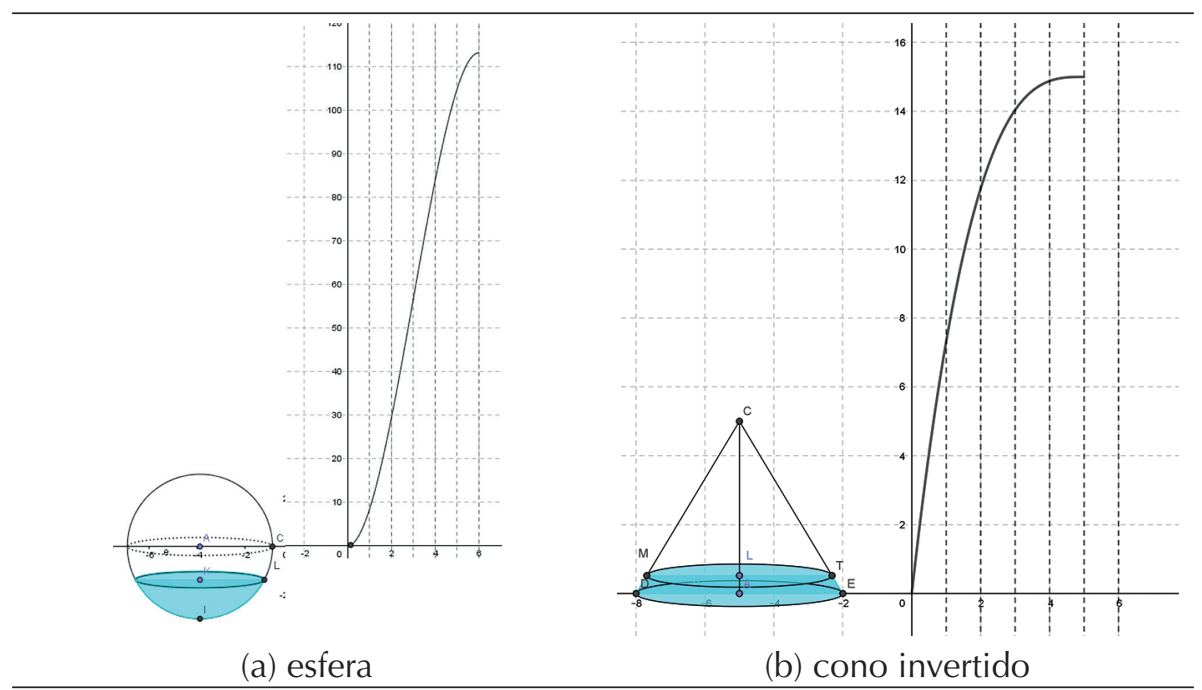

Figura 2. Gráficas de volumen vs altura.

El análisis de los datos incluyó, en primer lugar, la identificación de las secuencias de razonamiento de los estudiantes, las cuales constituyeron las unidades de análisis, posteriormente, en cada una de las secuencias se analizaron los diferentes estados del conocimiento alcanzado, los argumentos utilizados, y las posibles organizaciones de conocimientos. La representación gráfica de las secuencias de razonamiento permitió identificar visualmente los procesos de solución de la tarea y los argumentos usados para justificar los resultados. Además, cada gráfica dirigida fue elemento auxiliar para reconocer conexiones entre ideas y el posible impacto de la herramienta en la construcción de tales conexiones.

\section{Resultados}

Durante la primera sesión se pidió a los estudiantes considerar un cilindro con altura 6 y radio 2. La configuración dinámica inicial (Figura 3) tenía desactivadas las opciones para visualizar la gráfica y la ecuación de la función que modela el fenómeno. El instructor solicitó a los estudiantes mover el punto $P$, el cual controla la altura del líquido, de forma que ésta tomara valores específicos $(0.5,1,1.5,2$, etcétera), con la finalidad de que los estudiantes identificaran una correspondencia 
El rol de la tecnología en el desarrollo de entendimiento matemático vía la resolución de problemas

Fernando Barrera-Mora y Aarón Reyes-Rodríguez

entre cambios en la altura y en el volumen, es decir, que reconocieron cuáles son las cantidades que varían, y la relación entre esas variaciones. También se les solicitó calcular el volumen de líquido correspondiente a las alturas anteriores, para corroborar que los valores mostrados en la hoja dinámica correspondían al volumen del líquido contenido en el recipiente. La posibilidad de arrastrar el punto $P$, favoreció el que los estudiantes centraran la atención en la altura como variable.

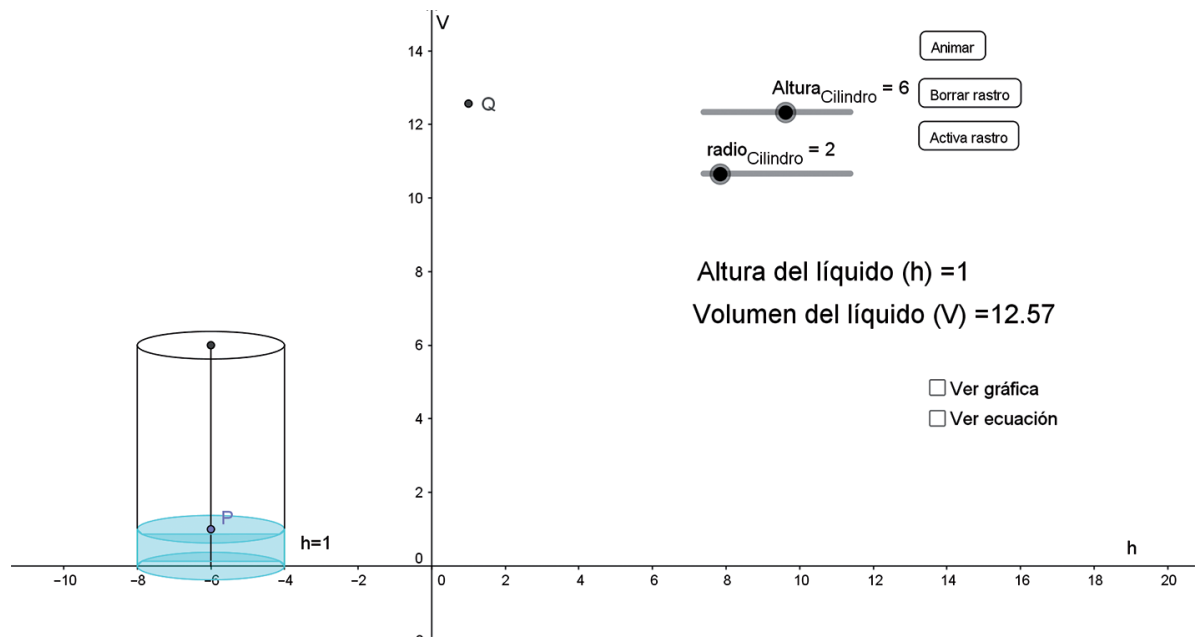

Figura 3. Llenado de recipientes cilíndricos.

Otro aspecto que se destacó durante las sesiones de trabajo fue centrar la atención en el comportamiento del punto $Q$, con el objetivo de relacionar las coordenadas de este punto con otros elementos de la configuración dinámica. Los estudiantes identificaron que la primera coordenada de $Q$ corresponde al valor de la altura, mientras que la segunda coordenada indica el valor del volumen. También se les pidió que analizaran el cambio en el volumen cuando la altura pasa de 0 a 1, de 1 a 2, y así sucesivamente. Posteriormente, se les pidió explorar lo que ocurre con otros incrementos constantes de la altura. Los estudiantes notaron que a un mismo incremento de la altura corresponden incrementos aproximadamente iguales del volumen. A partir de los datos indicados calcularon las tasas promedio de cambio y determinaron que estas tasas son aproximadamente iguales. A partir de eso conjeturaron que la tasa instantánea de cambio del volumen respecto de la altura debería ser constante y con un valor cercano a 12.57, que es una aproximación de 4ח. 
Posteriormente se pidió a los estudiantes que dieran movimiento al punto $P$ y observaran la trayectoria descrita por $Q$. Los estudiantes conjeturaron que este punto se mueve sobre una recta. Después de formular esta conjetura, seleccionaron las opciones "ver gráfica" y "ver ecuación" en la hoja dinámica. Ellos determinaron que el volumen varía de forma proporcional a la altura, ya que para calcular el volumen simplemente se multiplica el área de la base (una circunferencia) por la altura, así que la constante de proporcionalidad es igual al área del círculo que es base del cilindro, es decir, $4 \Pi \approx 12.57$ y por esta razón la ecuación de la recta que representa al volumen de este cilindro, como función de la altura, es $V=12.57 \mathrm{~h}$.

Un segundo momento del desarrollo de la actividad, con la configuración dinámica, consistió en variar el radio y la altura del cilindro. Al variar el radio del cilindro, los estudiantes comentaron que se modificaba la razón de cambio del volumen respecto de la altura, es decir, a menor radio del cilindro la variación del volumen por unidad de altura también es menor, y este resultado se ve reflejado en la pendiente de la recta que modela el Ilenado de ese recipiente. Es decir, a menor radio del cilindro le corresponde una recta con menor pendiente, ya que la pendiente de la recta corresponde al área de la base del cilindro.

Para analizar el volumen como función de la altura, en el caso de casquetes esféricos, al igual que en el caso de los cilindros, los estudiantes visualizaron el volumen para valores específicos de la altura. Analizaron, mediante incrementos uniformes de la altura del líquido, las tasas promedio de cambio del volumen para diferentes secciones transversales. En este caso hubo algunas complicaciones, debido a diferencias con el caso de los cilindros, las tasas promedio de cambio del volumen son variables, ya que dependen de la anchura de la sección transversal del recipiente. Para analizar las tasas promedio de cambio, los estudiantes sugirieron trazar líneas auxiliares paralelas al eje vertical con la finalidad de identificar, con mayor facilidad, los incrementos de volumen, debidos a incrementos constantes de la altura. Así, pudieron observar que a un mismo incremento de la altura le corresponde un mayor incremento del volumen en aquellas zonas del recipiente que son más "anchas". En este caso, los estudiantes sabían que las gráficas de volumen vs altura no estaban representadas por líneas rectas, ya que las tasas de cambio por cada incremento unitario de altura, no eran las mismas. Sin embargo, les fue difícil conjeturar el tipo de curva descrita por el punto $Q$ con la es- 
cala de medida estándar, ya que en esta escala la curvatura de la gráfica sólo se aprecia para puntos cercanos al origen. Esto motivó que hicieran uso del zoom de forma que la curvatura de la gráfica fuera evidente. Los estudiantes conjeturaron que la gráfica del volumen es una función cúbica, debido a que el volumen de una esfera de radio $R$ se relaciona con la expresión $\Pi \mathrm{R}^{3}$. Nos parece importante mencionar que para obtener la fórmula del volumen de un casquete esférico se requieren conocimientos de cálculo integral, por lo que es razonable que los estudiantes, quienes recién habían egresado de bachillerato, no hayan obtenido la fórmula que describe el volumen del casquete esférico como función de la altura, la cual es $V=\frac{\pi}{3} h^{2}(3 R-h)$. Los estudiantes mencionaron que la gráfica se parece mucho a una recta, porque las tasas de cambio son grandes.

Posteriormente, los estudiantes variaron el radio de la esfera y se dieron cuenta que a medida que éste se incrementaba, era más difícil visualizar curvatura en la gráfica del volumen. Adicionalmente, observaron que el volumen se incrementaba más rápidamente para los mismos incrementos constantes de la altura, esto en relación con esferas de menor radio.

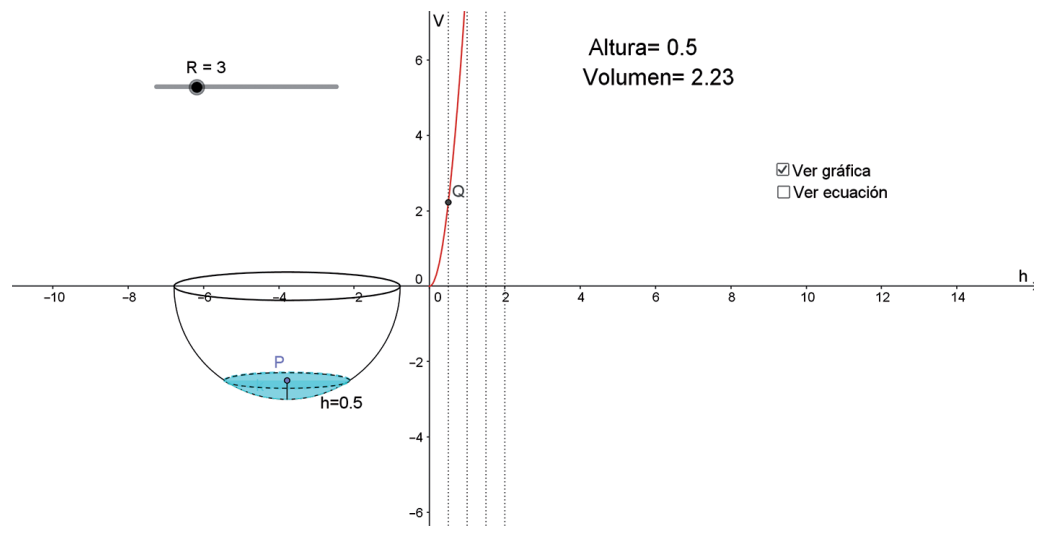

Figura 4. Llenado de casquetes esféricos.

El análisis del Ilenado de recipientes cónicos, se inició con un cono, con radio de la base igual a dos y una altura de cinco unidades. Los estudiantes, al igual que en el caso de los casquetes esféricos, propusieron utilizar líneas auxiliares para analizar la tasa promedio de cambio debida a diferentes incrementos constantes de la altura. En esta tarea algunos estudiantes conjeturaron que la curva que modela el volumen como función de la altura es una parábola. Esta observación fue motivada por 
la gráfica mostrada en la hoja dinámica. Sin embargo, otros estudiantes rechazaron esta conjetura argumentando que la gráfica debe estar representada por una función cúbica, ya que se trata de un volumen, pero otros estudiantes hicieron referencia al caso del cilindro, donde también se está modelando el volumen y no obstante, la ecuación de la gráfica es una función lineal. Es interesante notar que estas observaciones de los estudiantes pueden llevar a preguntas relevantes, desde el punto de vista matemático. Por ejemplo, ¿para qué secciones transversales de los recipientes, el volumen es una función cuadrática de la altura? El tratar de responder a preguntas parecidas ofrece oportunidades para poner en práctica los elementos fundamentales del pensamiento matemático.

Los estudiantes observaron que en el caso del cono, las tasas promedio de cambio son menores que para los casquetes esféricos, porque las secciones transversales del cono aumentan en menor medida que en el caso de los casquetes esféricos.

El uso de las líneas auxiliares fue de utilidad para el análisis de las tasas promedio de cambio, correspondientes a incrementos unitarios de la altura, ya que las intersecciones de la gráfica con las rectas auxiliares favorecieron a que los estudiantes identificaran rectas imaginarias que representan esas tasas. Los estudiantes notaron que a medida que el cono se hace más ancho, se incrementa la razón de cambio del volumen. $\mathrm{Al}$ aumentar el radio de la base del cono, los estudiantes identificaron que la gráfica del volumen se "adelgaza", lo cual implicaba que las tasas promedio de cambio se incrementaban, dado que el incremento del radio provocaba incrementos de las secciones transversales del cono.

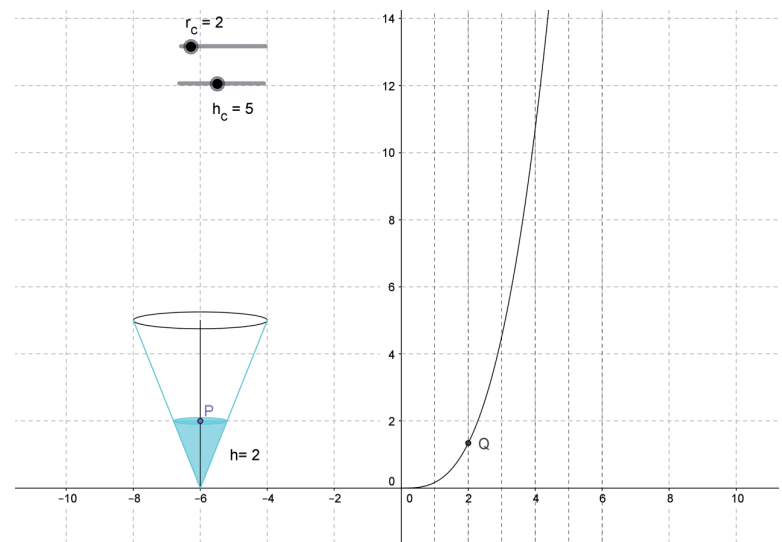

Figura 5. Llenado de un cono. 


\section{Análisis del comportamiento gráfico del volumen sin apoyo del profesor}

Equipo A (volumen vs altura en una esfera). El representante del equipo, Adrián, mencionó que el área del círculo está dada por la fórmula $\pi r^{2}$ y a partir de ahí dedujo, incorrectamente, que la fórmula del volumen de una esfera es $\pi r^{3}$, mencionó que el caso particular que consideraron en el equipo es de una esfera de diámetro uno. Entonces explicó que en primer lugar determinaron que cuando la altura del líquido es cero, también el volumen es cero. Cuando se ha llenado la mitad de la esfera, "la altura es un medio" y el volumen es un octavo de pi, es decir, $\pi(1 / 2)^{3}$. En este equipo pensaron en el volumen de una esfera de radio 1/2, más que en el volumen en un casquete esférico. El estudiante no se percata que la expresión $\pi(1 / 2)^{3}$ representaría el volumen total de una esfera de radio $1 / 2$, en caso de que la fórmula fuese correcta. A continuación marca el punto de coordenadas $(1 / 2, \pi / 8)$ y menciona que cuando la esfera está Ilena, su volumen es igual a $\pi$.

Más allá del cálculo incorrecto del volumen de los casquetes esféricos, los estudiantes centraron la atención solamente en tres puntos particulares para bosquejar la gráfica: cuando la altura es cero, 1/2 y 1 . Sin embargo, se produjo un bosquejo poco preciso en el que no consideraron el problema de llenado del recipiente con forma de casquete esférico, ni tomaron en cuenta la simetría que debería tener la curva, debida a que las dos mitades de la esfera tienen comportamientos parecidos, uno con una tendencia creciente de las tasas de cambio, mientras que el otro con una tendencia decreciente. A pesar de lo anterior, en este equipo identificaron, de forma implícita, un punto de inflexión de la gráfica en $(1 / 2, \pi / 8)$ y un cambio de concavidad en la gráfica, pero no mencionaron las razones de éste (ver gráfica a la derecha en la Figura 5). Otro aspecto que es importante destacar, es que los estudiantes no utilizaron líneas verticales sobre una equipartición del dominio de la función para analizar las diferentes tasas promedio de cambio como ocurrió con las experiencias previas. 


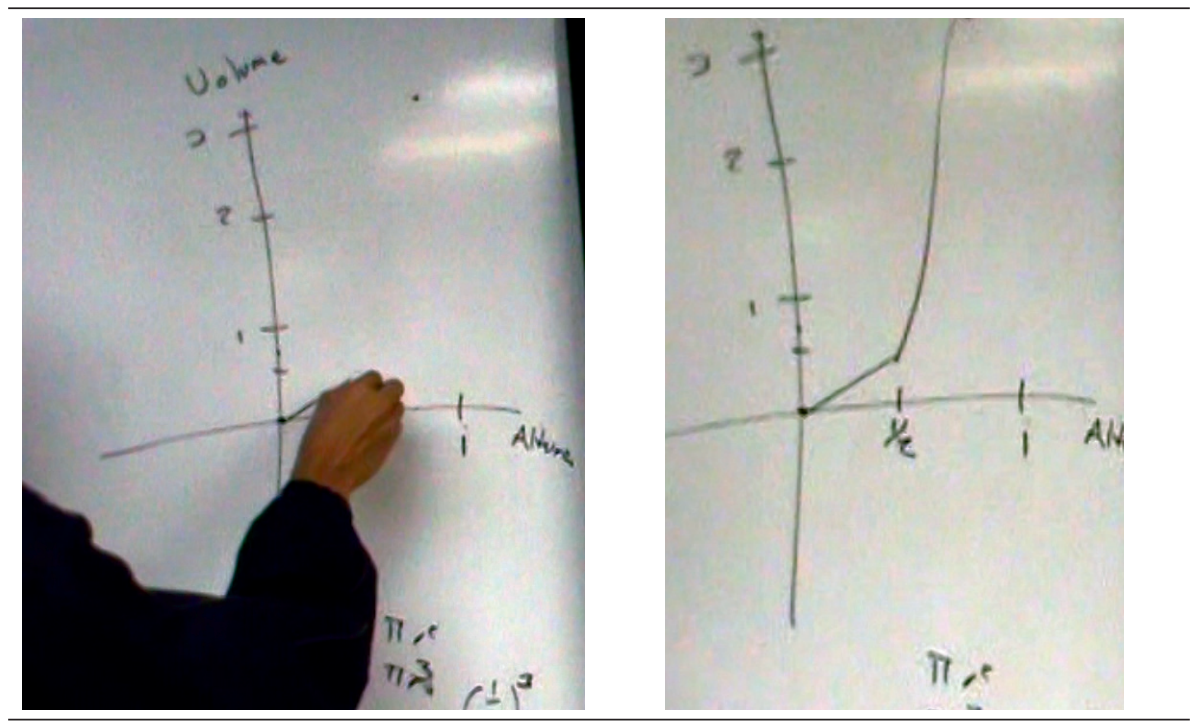

Figura 6. Bosquejo de la gráfica de volumen vs altura, recipiente esférico (equipo $\mathrm{A}$ ).

Equipo B (volumen vs altura en una esfera). La representante del equipo, Antonia, explicó que en el eje horizontal se marcaba la altura y en el vertical el volumen, lo cual es indicador de una identificación correcta de la variable y la función. Los estudiantes identificaron que cuando la altura es cero, el volumen de la esfera también es cero y esta información se representa con el origen del sistema coordenado. Luego, conforme la altura aumenta, también lo hace el volumen hasta que éste abarque la mitad de la esfera y en la segunda mitad de ésta, el volumen irá subiendo más lentamente hasta alcanzar un valor de $\Pi$, porque ellos consideraron que el volumen de la esfera se calcula mediante la fórmula $\Pi r^{3}$. Conceptualmente identifican máximos, aunque prevalece el error para medir el volumen a una altura $h$. Los estudiantes del equipo $\mathrm{B}$, al igual que los estudiantes del equipo $A$, también cometieron el error de calcular, mediante una fórmula incorrecta, el volumen de una esfera de radio 1 y no el de una esfera de diámetro 1 . En este equipo, aunque se dieron cuenta que la gráfica del volumen tiene comportamientos distintos en los intervalos $[0,1 / 2]$ y $[1 / 2,1]$, expresado en el siguiente comentario de Ana durante la exposición plenaria: "y posteriormente [después que se llenó la mitad de la esfera] sí vi, como decía Mario, [el volumen] va ir subiendo ya más poco, iba ir subiendo más lentamente [la tasa ins- 
El rol de la tecnología en el desarrollo de entendimiento matemático vía la resolución de problemas

Fernando Barrera-Mora y Aarón Reyes-Rodríguez

tantánea de cambio disminuye]", no supieron representar gráficamente esta diferencia (ver gráfica de la derecha en la Figura 7).

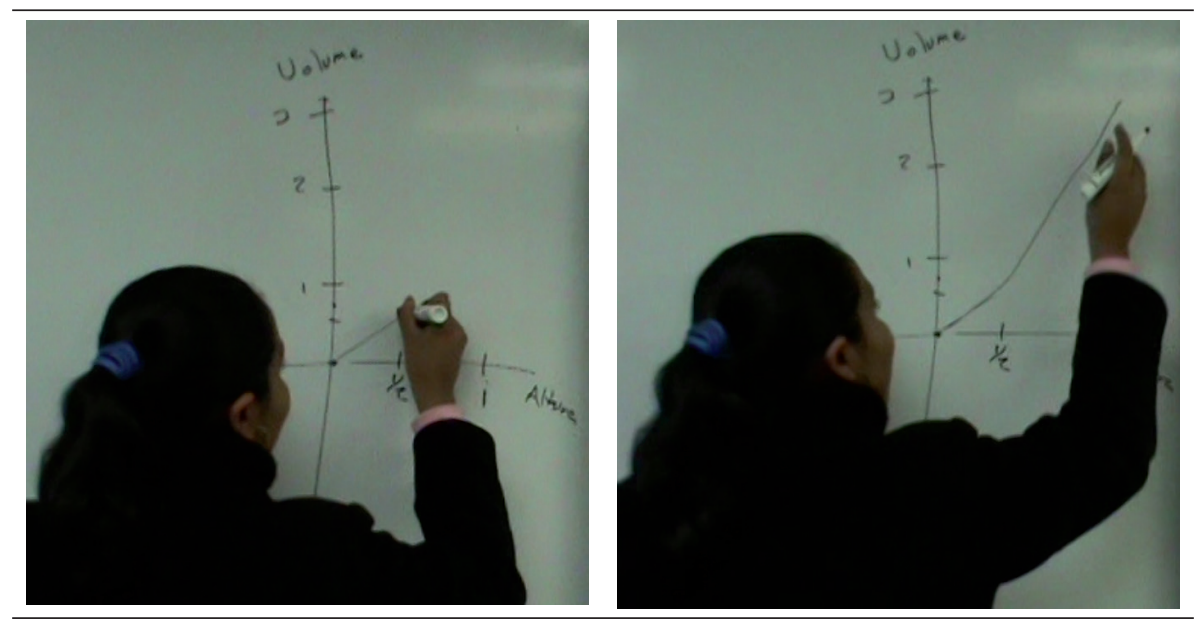

Figura 7. Bosquejo de la gráfica de volumen vs altura, recipiente esférico (equipo B).

Equipo C (volumen vs altura en una esfera). El representante del equipo, Oscar, comentó que se dieron cuenta de que cuando la altura es muy pequeña, la gráfica parece una recta porque el volumen va aumentando "muy poquito". Pero conforme la altura se va aproximando al valor del radio de la esfera, el volumen aumenta cada vez más ("mucho, mucho mucho"). Entonces, cuando se ha llenado la mitad de la esfera se ha alcanzado el máximo de crecimiento. El estudiante recuperó dos de las observaciones realizadas durante la primera parte de las tareas. Estas son: (i) que la tasa de cambio no es contante si la sección transversal del recipiente varía y que (ii) es mayor en las partes "más anchas del recipiente".

"A partir de que se llena la mitad de la esfera, el volumen empieza a crecer cada vez más lento, más lento, hasta que otra vez llega a ser como una línea. Y ya lo constatamos después viendo que el volumen está dado por cuatro tercios de п por $r$ al cubo, y $r$ es cúbica, entonces tiene que salir algo así, una cúbica". El estudiante relacionó cambios pequeños en el volumen con la gráfica de una recta, sin embargo, como se analizó en el caso del Ilenado de cilindros, una gráfica recta indica tasas de cambio constantes, que corresponden a secciones transversales 
iguales. Tal vez lo que el estudiante quiso decir es que cuando la altura es muy cercana a cero, las secciones transversales se modifican poco y por ello la gráfica del volumen vs altura se puede aproximar con una recta. Los estudiantes de este equipo también identificaron la existencia de un punto de inflexión (Figura 8) y un cambio de concavidad de la gráfica asociado a comportamientos diferentes en lo que respecta a las tasas instantáneas de cambio en el volumen, después de que se ha llenado la mitad de la esfera.

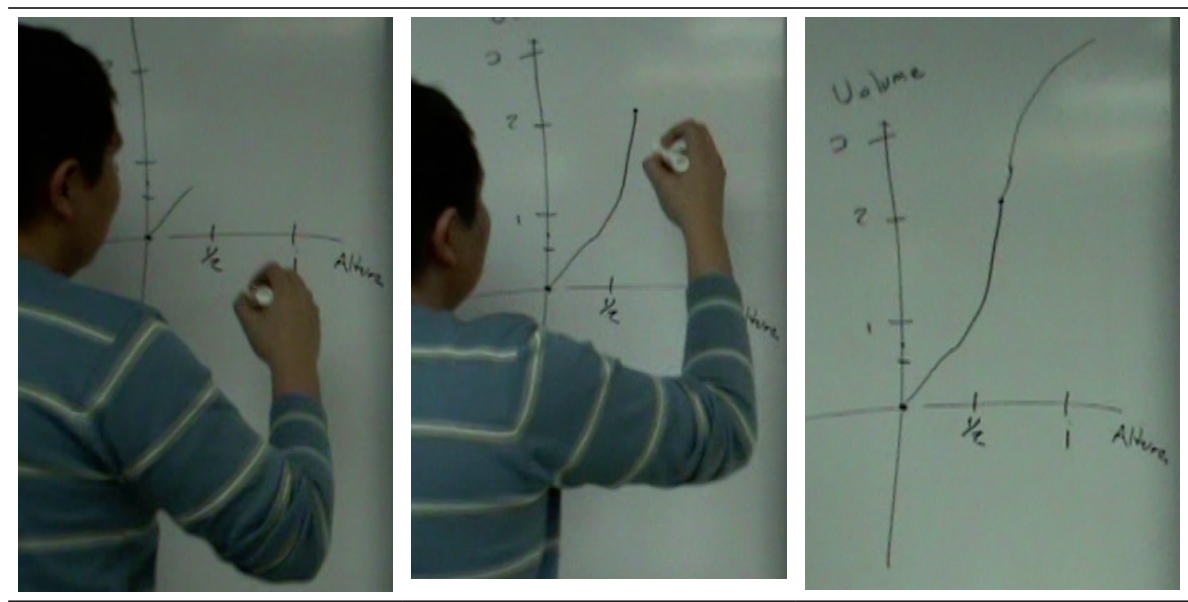

Figura 8. Bosquejo de la gráfica de volumen vs altura, recipiente esférico (equipo $\mathrm{C}$ ).

Equipo D (volumen vs altura en un cono invertido). El representante del equipo, Gerardo, mencionó que en la base del cono el crecimiento del volumen es grande, y por esa razón la gráfica de volumen vs altura tiene la forma de una recta, después, dado que la sección transversal disminuye, también disminuye la tasa de cambio del volumen, y por esta razón la gráfica del volumen se puede aproximar también con una recta, pero con pendiente mayor que la primera que trazó (gráfica de la izquierda en la Figura 9). Otra integrante del equipo, Sara, argumentó que la gráfica tiene la forma que ellos propusieron porque "Este es un punto [señalando la gráfica] que sube, que sube muy rápido [dando a entender que la tasa de cambio cerca de la base es grande]...ahora sí que el volumen sube muy rápido, pero ya de aquí [señala la mitad de la altura] va disminuyendo". Después de analizar su explicación, Sara 
decidió que el bosquejo de la gráfica debía ser diferente, primero trazó un bosquejo similar al de media parábola el cual indicó que representa el crecimiento rápido inicial del volumen (gráfica central en la Figura 9) y posteriormente indicó que, dado que la tasa de cambio era pequeña a partir de la mitad del cono, la gráfica de volumen vs altura se podía aproximar mediante una recta: "entonces como va más lentamente [la tasa de cambio del volumen] entonces sería como una especie de recta" (gráfica de la derecha en la Figura 9). En ambas aproximaciones, los integrantes de este equipo identificaron una variación en la tasa instantánea de cambio, que representaron mediante el punto donde se unen las dos rectas que dibujaron, pero no fueron capaces de identificar que la disminución de la razón de cambio es uniforme a partir de la base del cono, y que este cambio no es brusco, como lo indica la primera gráfica que ellos dibujaron.
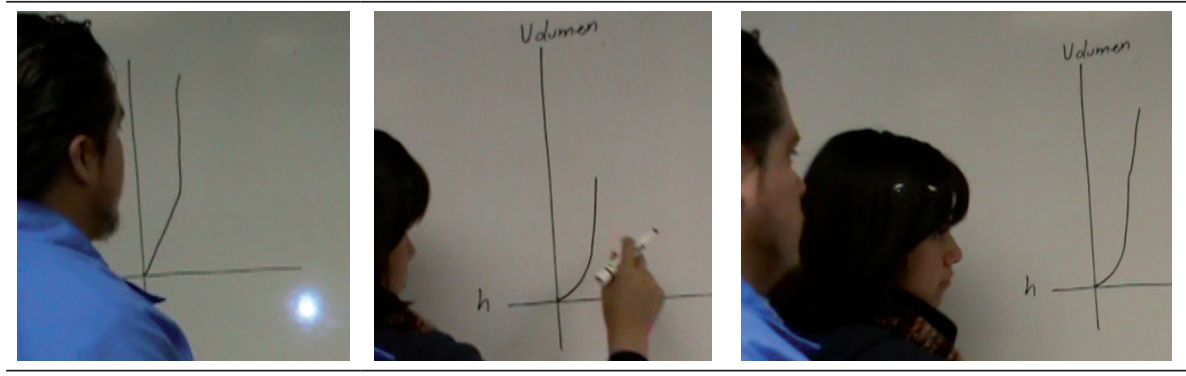

Figura 9. Bosquejo de la gráfica de volumen vs altura, recipiente cónico (equipo D)

Equipo $\mathrm{E}$ (volumen vs altura en un cono invertido). El representante del equipo, Fabián, inicialmente indicó que la gráfica de volumen vs altura es similar a media parábola (gráfica de la izquierda en la Figura 10), pero posteriormente ajustó la gráfica a una que tiene una forma de $S$ en la que se identifica un cambio de concavidad (gráfica de la derecha en la Figura 10). El estudiante argumentó que la gráfica es así, debido a que "aquí [cerca de la base del cono], conforme va aumentado la altura, el radio sigue siendo grande y, entonces, el volumen es grande, y aquí el radio se va haciendo más pequeñito [señala desde la mitad de la altura hasta el vértice del cono] y entonces va aumentado el volumen más lento". Al parecer del estudiante, este cambio de comportamiento lo llevó a concluir que la gráfica debería tener un cambio de concavidad, pero 
no se dio cuenta que este cambio, que el notó en la tasa de cambio, se presenta de manera uniforme desde la base del cono, a diferencia del caso de la esfera, donde la tasa instantánea de cambio aumenta progresivamente y luego disminuye de la misma forma a partir de que se llena la mitad de la esfera.

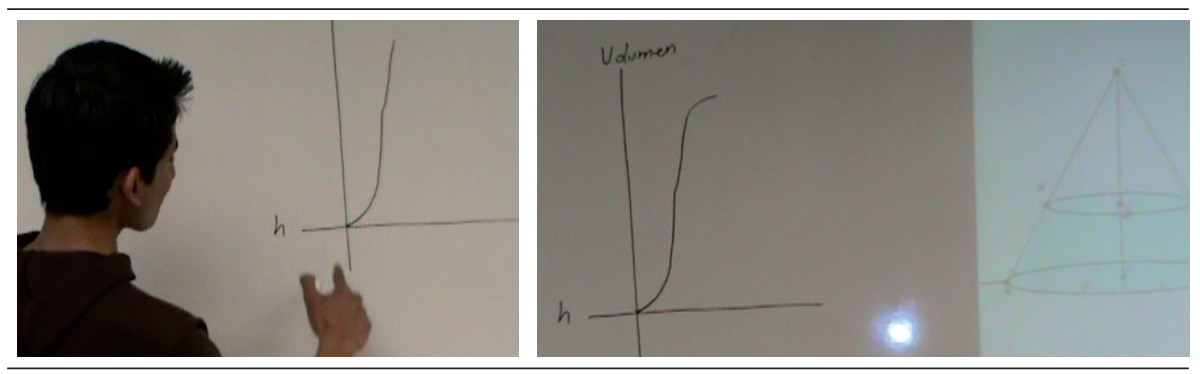

Figura 10. Bosquejo de la gráfica de volumen vs altura, recipiente cónico (equipo E).

Equipo $\mathrm{F}$ (volumen vs altura en un cono invertido). En este equipo hubo una discrepancia de opiniones, ya que la representante del equipo, Mara, dibujó una gráfica que tiene un cambio de concavidad (ver gráfica de la izquierda en la Figura 11). Durante el trazo del bosquejo comentó: "Nosotros pensamos que va así porque de aquí [la base del cono] empieza grande [la tasa de cambio] y luego disminuye". Sin embargo, otro integrante del equipo, Marco, mencionó que no estaba de acuerdo con la gráfica de Mara y realizó otra propuesta (ver gráfica de la derecha, con trazo grueso, en la Figura 11), la cual es la gráfica correcta que modela el comportamiento del volumen como función de la altura para el cono invertido. Marco mencionó que: "No...bueno, es que como la figura va abriendo de una forma, este, el volumen va elevándose mucho y va disminuyendo [la tasa instantánea de cambio] conforme la altura va avanzando, entonces no abriría así [como la dibujó Mara] no abriría de esta forma, sino que tendría esta forma" (gráfica de la derecha, en trazo grueso, de la Figura 11). En este caso Marco identificó, de forma correcta, que la variación de la tasa de cambio es continua a través de todo el dominio de la función, y por esta razón no hay en la gráfica de volumen vs altura, un punto de inflexión, como sí ocurre en la gráfica propuesta por Mara. En este episodio se puede observar que la interacción entre los integrantes del equipo llevó a que 
se exhibieran diferentes puntos de vista y que se llegara a un acuerdo con base en la argumentación.

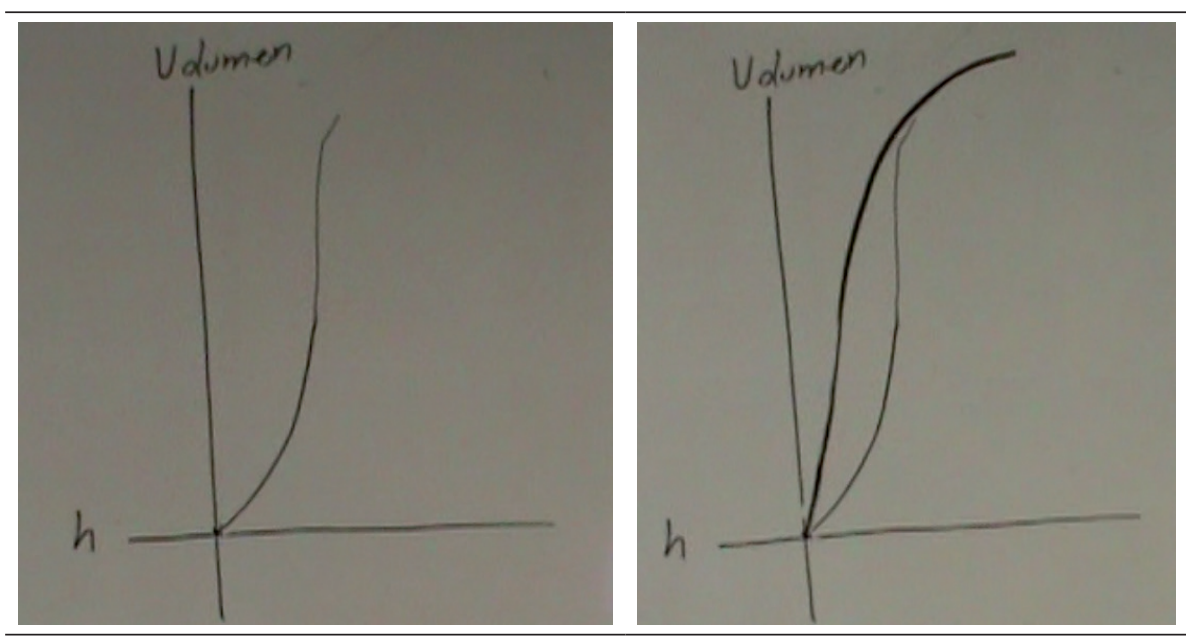

Figura 11. Bosquejo de la gráfica de volumen vs altura, recipiente cónico (equipo E).

\section{Discusión y conclusiones}

En este trabajo, a diferencia de lo revisado en la literatura, los estudiantes no tuvieron confusión respecto de cuál es la variable y cuál es la función, ya que las características de la herramienta permitieron a los estudiantes identificar la diferencia entre variable y función. Esto en relación con los puntos que se manipulan directamente por el usuario, y aquellos que resultan en este proceso. Además, el uso de la tecnología les permitió identificar de una forma global el comportamiento gráfico del fenómeno y se evitó la traslación de formas, identificada por Hitt-Espinosa (1995), lo cual aparece cuando no se les ofrecen a los estudiantes medios que les permitan visualizar los procesos de variación, sino solo imaginarlos.

Observamos que el uso del software cambia el tipo de acciones y procesos cognitivos que los estudiantes llevan a cabo en un ambiente de papel y lápiz. Por ejemplo, de acuerdo con Carlson, Larsen y Lesh (2003), se han identificado seis categorías de acciones mentales que los estudiantes llevan a cabo cuando representan e interpretan gráficamente 
un fenómeno que involucra variación y cambio en ambientes de papel y lápiz. Estas seis categorías de imágenes mentales son: (1) una imagen de dos variables que cambian simultáneamente, (2) una imagen coordinada general de cómo las variables cambian, una respecto de otra (crece o decrece), (3) una imagen del cambio de la función para incrementos fijos de la variable, (4) una imagen de la tasa de cambio para intervalos, (5) una imagen de la tasa de cambio instantánea en todo el dominio de la función y (6) una imagen de los cambios en la tasa de cambio, creciente o decreciente. En el caso del uso de la tecnología, el estudiante manipula y visualiza, y no sólo imagina la variación y la relación entre las variables. Específicamente, el estudiante puede manipular el incremento de la variable y observar el correspondiente incremento del volumen. En el caso del aspecto (c), el uso de líneas verticales es un apoyo que puede ayudar a los estudiantes a visualizar el cambio de la función para incrementos fijos de la variable. Las representaciones gráficas tienen poco impacto en la forma en que los estudiantes establecen conjeturas, con respecto a las ecuaciones que modelan esas gráficas, ya que las gráficas de funciones cúbicas, localmente tienen parecido a funciones lineales o cuadráticas. El zoom es una herramienta útil para que los estudiantes puedan identificar con mayor precisión la curvatura de las gráficas de volumen.

Tabla 1. Acciones mentales desarrolladas por los estudiantes.

\begin{tabular}{|c|c|c|c|}
\hline Problema & Equipo & Acciones mentales & Impacto del uso de GeoGebra \\
\hline \multirow{3}{*}{ Esfera } & A & $1,2,4$ & \multirow{6}{*}{$\begin{array}{l}\text { La herramienta permitió a los estu- } \\
\text { diantes visualizar la coordinación en } \\
\text { el cambio entre la función y la varia- } \\
\text { ble. La configuración dinámica apo- } \\
\text { yó la visualización del cambio en las } \\
\text { secciones transversales del recipiente. } \\
\text { Esto último favoreció, en algunos ca- } \\
\text { sos, identificar variaciones en las ta- } \\
\text { sas de cambio. }\end{array}$} \\
\hline & $B$ & $1,2,4$ & \\
\hline & $\mathrm{C}$ & $1,2,4,5,6$ & \\
\hline \multirow[b]{3}{*}{ Cono } & $\mathrm{D}$ & $1,2,4$ & \\
\hline & $\mathrm{E}$ & $1,2,4,5,6$ & \\
\hline & $\mathrm{F}$ & $1,2,4,5,6$ & \\
\hline
\end{tabular}

(1) Imagen de dos variables que cambian simultáneamente, (2) imagen coordinada general de cómo cambian las variables, una respecto de otra (crece o decrece), (3) imagen del cambio de la función para incrementos fijos de la variable, (4) imagen de la tasa de cambio para intervalos, (5) imagen de la tasa de cambio instantánea en todo el dominio de la función y (6) imagen de los cambios en la tasa de cambio, si es creciente o decreciente. 
El rol de la tecnología en el desarrollo de entendimiento matemático vía la resolución de problemas

Fernando Barrera-Mora y Aarón Reyes-Rodríguez

Todos los estudiantes mostraron las acciones mentales del tipo 1 y 2 , ya que identificaron que el volumen es una función creciente de la altura. En el caso del equipo E, el error en la representación gráfica pudiera deberse a que, al pensar en una parábola, imaginan la imagen prototípica (cóncava hacía arriba), pero posteriormente se notó cómo el estudiante entendió que la tasa de cambio disminuye. De los resultados de la tabla, se puede observar el impacto que tuvo la fase guiada en el trabajo por equipo de los estudiantes. Por ejemplo, que la forma recipiente determina la razón de cambio, particularmente se destaca la relevancia de las secciones transversales.

Existen algunas limitantes con el uso de la herramienta. Por ejemplo, los estudiantes se formaron la idea de que, cambios grandes en la sección transversal de los recipientes producen gráficas que parecen rectas y este esquema mental lo aplicaron para resolver la tarea sin el apoyo del profesor. Es importante entonces, evaluar el tipo de entendimiento generado por los estudiantes, esto debido a que el profesor puede pensar que se desarrolló cierto tipo de esquema, cuando en realidad el esquema formado es otro, completamente diferente al supuesto. Tal vez se requiere mayor reflexión en torno al significado de ciertas palabras o enunciados que precisen su significado en un contexto matemático.

En los casos analizados, los estudiantes mostraron elementos que llevan a conjeturar que el uso de herramientas como GeoGebra, les permite incorporar a su imagen conceptual de función, representaciones gráficas de funciones definidas por tramos, es decir, aquellas que están definidas por dos o más reglas de asignación. Este es el caso de aquellas funciones que emergen, en el proceso de llenado de casquetes de las esferas. Los resultados que estamos presentando contrastan con los que se reportan de otras investigaciones citadas por Hitt (1995, p. 65).

Existen expresiones que se deben precisar durante el proceso de discusión de las ideas, debido a que esto puede llevar a los estudiantes a realizar interpretaciones en forma errónea, como ocurrió en este trabajo. Particularmente, en los casos en que las funciones que modelan el volumen se pueden aproximar por funciones lineales en intervalos grandes.

Los estudiantes determinaron de forma correcta si las funciones eran crecientes o decrecientes, pero hace falta poner atención en el proceso que les permita determinar la concavidad correcta de las gráficas, así como los puntos de inflexión y su significado en términos de crecimiento. En este tipo de actividades los estudiantes deben coordinar al menos 
dos tipos de información, la que se refiere a la gráfica en sí, que modela al volumen como función de la altura del líquido, así como también deben articular la información referente a las tasas instantáneas de cambio, lo cual no ocurre en las tareas comunes sobre graficación.

Una observación efectuada durante el desarrollo de las tareas, es que el estado actual de GeoGebra tiene limitaciones para representar, en la misma pantalla, una gráfica que ilustre cambio de volumen con el cambio de altura, o viceversa. Con esta limitante, el abordar tareas en donde se desea maximizar volúmenes, con ciertas restricciones, requiere del uso de expresiones algebraicas. Esto contrasta con lo que ocurre al abordar problemas para maximizar áreas sin el uso de un modelo algebraico como se ha hecho en otros trabajos (Santos-Trigo, 2015; Barrera-Mora y Reyes-Rodriguez, 2016). Se espera que el uso sistemático de la tecnología, en el desarrollo de tareas de instrucción, proporcione fundamentos para entender los procesos de variación y cambio, lo cual pudiese permitir a los estudiantes analizar otros fenómenos que no se pueden modelizar con el uso de la tecnología disponible, como GeoGebra. Por ejemplo, abordar tareas en las que los recipientes tengan formas delimitadas por objetos geométricos más generales que rectas o circunferencias.

Finalmente, estamos de acuerdo con Drijvers (2015) en que la integración de la tecnología en los salones de clase no es la panacea que resolverá los problemas de aprendizaje. El profesor es y seguirá siendo un actor fundamental, ya que es él quien propone tareas que aprovechan los recursos didácticos que ofrecen las diferentes herramientas y organiza el ambiente de instrucción, resalta o sugiere rutas para avanzar en el proceso de solución de los problemas y promueve procesos de reflexión que serán útiles para generar entendimiento matemático.

\section{Reconocimientos}

Agradecemos el apoyo brindado para la realización de este trabajo a través de los proyecto Conacyt-168543 (México), y del Plan Nacional I+D+I del MCIN (España) EDU2015-65270-R y EDU2017-84276-R. 
El rol de la tecnología en el desarrollo de entendimiento matemático vía la resolución de problemas

Fernando Barrera-Mora y Aarón Reyes-Rodríguez

\section{Referencias}

Barrera-Mora, F., \& Reyes-Rodriguez, A. (2016). Designing technology-based tasks for enhancing mathematical understanding through problem solving. In L. Uden, D. Liberona and B. Feldmann (Eds.), Learning technology for education in cloud: The changing face of education (pp. 183-192). Switzerland: Springer.

Berger, W. (2014). A more beautiful question: The power of inquiry to spark breakthrough ideas. New York, NY: Bloomsbury.

Brown, C. \& Borko, H. (1992). Becoming a mathematics teacher. In D. A. Grouws (Ed.), Handbook of research on mathematics teaching and learning (pp. 209-239). New York: Macmillan.

Carlson, M. P., Jacobs, S., Coe, E., Larsen, S., \& Hsu, E. (2002). Applying covariational reasoning while modeling dynamic events: A framework and a study. Journal for Research in Mathematics Education, 33(5), 352-378.

Carlson, M. P., Larsen, S., \& Lesh, R. A. (2003). Integrating a models and modeling perspective with existing research and practice. In R. A. Lesh and H. Doerr (Eds.), Beyond constructivism: Models and modeling perspectives on mathematics problem solving, learning and teaching (pp. 465-478). Mahwah, NJ: Lawrence Erlbaum Associates.

Chimhande, T., Naidoo, A., \& Stols, G. (2017): An analysis of Grade 11 learners' levels of understanding of functions in terms of APOS theory. Africa Education Review, 14(34), 1-19.

Cobb, P., Wood, T., Yackel, E., Nichols, J., Wheatley, G., Trigatti, B., \& Perlwitz, M. (1991). Assessment of a problem-centered second grade mathematics project. Journal for Research in Mathematics Education, 22, 3-29.

de Beer, H., Gravemeijer, K., \& van Eijck, M, (2015). Discrete and continuous reasoning about change in primary school classrooms. ZDM Mathematics Education, 47, 981996.

de Beer, H., Gravemeijer, K., \& van Eijck, M. (2017). A proposed local instruction theory for teaching instantaneous speed in grade five. The Mathematics Enthusiast, 14(1), Article 24. Recuperado el 16 de marzo de 2017 de http://scholarworks.umt.edu/tme/ vol14/iss $1 / 24$

De Faria-Campos, E. (2004). Funciones embotelladas. En L. Díaz (Ed.), Acta Latinoamericana de Matemática Educativa (pp. 584-589). México: Comité Latinoamericano de Matemática Educativa.

Donald, M. (1993). Origins of the modern mind. Cambridge, Massachusetts: Harvard University Press.

Drijvers, P. (2015). Digital technology in mathematics education: Why it works (or doesn't). In S. J. Cho (Ed.), Selected regular lectures from the 12th International Congress on Mathematical Education (pp. 135-151). Cham: Springer.

Glaister, E. \& Glaister, P. (2006). Introducing conics without eccentricity. International Journal of Mathematical Education in Science and Technology, 37(2), 235-245.

Heid, M. K., Lunt, J., Portnoy, N., \& Zembat, I. O. (2006). Ways in which prospective secondary mathematics teachers deal with mathematical complexity. In S. Alatorre, 
El rol de la tecnología en el desarrollo de entendimiento matemático vía la resolución de problemas

Fernando Barrera-Mora y Aarón Reyes-Rodríguez

J. L. Cortina, M. Sáiz \& A. Méndez (Eds.), Proceedings of the 28th annual meeting of the North American Chapter of the International Group for the Psychology of Mathematics Education (Vol. 2, pp. 2-9). Mérida, Mexico: PME-NA.

Hitt-Espinosa, F. (1995). Intuición primera versus pensamiento analítico: dificultades en el paso de una representación gráfica a un contexto real y viceversa. Educación Matemática, 7(1), 63-75.

Johnson, H. L. (2012). Reasoning about quantities involved in rate of change as varying simultaneously and independently. In R. Mayes \& L. L. Hatfield (Eds.), Quantitative reasoning and mathematical modeling: A driver for STEM integrated education and teaching in context (Vol. 2, pp. 39-53). Laramie, WY: University of Wyoming College of Education.

Johnson, H. L. (2013). Designing Covariation Tasks to Support Students' Reasoning about Quantities involved in Rate of Change. STEM Faculty Presentations. Paper 45. Recuperado el 16 de marzo de 2016 de http://source.ucdenver.edu/stem_presentations $/ 45$

Kaput, J. J., \& Schorr, R. Y. (2008). Changing representational infrastructures changes most everything. In G. W. Blume \& M. K. Heid (Eds.), Research on technology and the teaching and learning of mathematics: Cases and Perspectives (Vol. 2, pp. 211 253). Charlotte, NC: Information Age Publishing.

Kindle, J. H. (1970). Teoría y problemas de geometría analítica plana y del espacio (Trad. Luis Gutiérrez Díez y Ángel Gutiérrez Vázquez). México: McGraw-Hill.

Koehler, M. J. \& P. Mishra (2009). What is technological pedagogical content knowledge? Contemporary Issues in Technology and Teacher Education, 9(1), pp.60-70.

Lehmann, C. (1990). Geometría Analítica (Díaz, R. Trad.). México: Limusa. (Primera edición 1942).

Lithner, J. (2008). A research framework for creative and imitative reasoning. Educational Studies in Mathematics, 67(3), 255-276.

Mishra, P. \& Koehler, M. (2006). Technological Pedagogical Content Knowledge: A new framework for teacher knowledge. Teachers College Record, 108 (6), 1017-1054.

Moreno-Armella, L. (1996). Mathematics: a historical and didactic perspective. International Journal of Mathematical Education in Science and Technology, 27(5), 633639.

Moreno-Armella, L. (2002). Fundamentación cognitiva del currículo matemático. En Ministerio de Educación Nacional (Ed.), Memorias del Seminario Nacional Formación de Docentes sobre el Uso de Nuevas Tecnologías en el Aula de Matemáticas (pp. 4066). Bogotá, Colombia: Ministerio de Educación Nacional.

Moreno-Armella, L., \& Santos-Trigo, M. (2008). Democratic access and use of powerful mathematics in an emerging country. In L. D. English (Ed.), Handbook of International Research in Mathematics Education, 2nd Edition (pp. 319-351). New York: Taylor \& Francis.

Moreno-Armella, L., \& Santos-Trigo, M. (2015). The use of digital technology in mathematical practices. In L. D. English and D. Kirshner (Eds.), Handbook of International Research in Mathematics Education, 3rd Edition (pp. 595-616). New York: Roudledge. Moreno-Armella, L., Hegedus, S. J., \& Kaput, J. J. (2008). From static to dynamic mathe- 
El rol de la tecnología en el desarrollo de entendimiento matemático vía la resolución de problemas

Fernando Barrera-Mora y Aarón Reyes-Rodríguez

matics: historical and representational perspectives. Educational Studies in Mathematics, 68, 99-111.

National Council of Teachers of Mathematics (NCTM) (2010) Mathematics Curriculum. Issues, Trends, and Future Directions, edited by Reys, B.J., Reys, R.E. and Rubenstein, R., The Council, Reston, VA.

Pea, R. D. (1987). Cognitive technologies for mathematics education. En A. Schoenfeld (Ed.), Cognitive Science and Mathematics Education (pp. 89-122). Hillsdale: Erlbaum.

Reyes-Rodríguez, A., Vargas-Alejo, V., Cristobal-Escalante, C., y Soberanis-Cruz, V. (2015). Formas de razonamiento que emergen al resolver problemas de máximos y mínimos con un SGD. Epsilon. Revista de Educación Matemática, 91, 7-24.

Santos-Trigo, M. (2007). La resolución de problemas matemáticos: fundamentos cognitivos. Mexico: Trillas.

Santos-Trigo, M. (2014). Problem solving in mathematics education. In S. Lerman (ed.), Encyclopedia of Mathematics Education (pp. 496-501). New York: Springer.

Santos-Trigo, M. (2015). La construcción de modelos dinámicos en el estudio de fenómenos de cambio o variación y la resolución de problemas. En C. Azcárate, M. Camacho-Machín, M. T. González y M. Moreno (Coords.), Didáctica del Análisis Matemático: una revisión de las investigaciones sobre su enseñanza y aprendizaje en el contexto de la SEIEM (pp. 163-177). La Laguna: Universidad de la Laguna.

Santos-Trigo, M. (2018). Problematizar los contenidos y la tecnología digital. C2 Ciencia y cultura. Recuperado de http://www.revistac2.com/problematizar-los-contenidos/ el 26 de julio de 2018.

Santos-Trigo, M. \& Moreno-Armella, L (2016). The Use of Digital Technology to Frame and Foster Learners' Problem-Solving Experiences. In P. Felmer, E. Pehkonen \& J. Kilpatrick (Eds.), Posing and Solving Mathematical Problems. Advances and new perspectives (pp. 189-207). Switzerland: Springer.

Sarama, J., \& Clements, D.H. (2009). Early childhood mathematics education research: Learning trajectories for young children. New York: Routledge.

Schoenfeld, A. H. (1992). Learning to think mathematically: Problem solving, metacognition, and sense-making in mathematics. In D. Grouws (Ed.), Handbook for Research on Mathematics Teaching and Learning (pp. 334-370). New York: MacMillan.

Shell Centre for Mathematical Education [SCME] (1985). The language of functions and graphs: An examination module for secondary schools. Manchester, U.K.: Joint Matriculation Board.

Shulman, L. (1986). Those who understand: Knowledge growth in teaching. Educational Researcher, 15(2), 4-14.

Simon, M. A. (1994). Learning mathematics and learning to teach: Learning cycles in mathematics teacher education. Educational studies in mathematics, 26(1), 71-94.

Stein, M. K. \& Smith M. S. (1998). Mathematical tasks as a framework for reflection: From research to practice. Mathematics Teaching in the Middle School, 3, 268-275.

Tabach, M. (2011). A mathematics teacher's practice in a technological environment: A case study analysis using two complementary theories. Technology, Knowledge and Learning, 16(3), pp. 247-265. 
El rol de la tecnología en el desarrollo de entendimiento matemático vía la resolución de problemas

Fernando Barrera-Mora y Aarón Reyes-Rodríguez

Thompson, P. W., Byerley, C., \& Hatfield, N. (2013). A conceptual approach to calculus made possible by technology. Computers in the School, 30, 124-147.

Vygotsky, L. S. (1962). Thought and language. Cambridge, MA: MIT Press.

Vygotsky, L. S. (1978). Mind in society: The development of the higher psychological processes. Cambridge, MA: Harvard University Press.

Wertsch, J. V. (1993). Voices of the Mind: A Sociocultural Approach to Mediated Action. Cambridge, Massachusetts: Harvard University Press. 
